\title{
Study ON Remote Medical Monitoring System Based ON MSP430 AND CC2530
}

\author{
SONG Aijuan, SI Guangyuan, GU Qiongchan \\ School of Control Engineering, Northeastern University at Qinhuangdao, Qinhuangdao, China \\ songaijuan@163.com
}

Keywords: Remote Medical Monitoring; MSP430; CC2530; ZigBee

\begin{abstract}
The paper deals with the monitoring system based on ZigBee wireless sensor network technique in details. The system adopts single-chippedMSP430 and CC2530. The paper mainly introduces hardware circuit design of processor module, data acquisition module, and wireless communication module. The monitoring software of the host computer is developed by LabVIEW. Experiments prove that the system has good stability and security. It has very high practical value and promoting function in telemetrically monitoring field in the future, such as the pulse, blood pressure, blood oxygen saturation and electrocardiogram.
\end{abstract}

\section{Introduction}

Remote Medical Monitoring System, also known as remote patient or healthcare monitoring, expands the usefulness of telemedicine in recent year. At first, it treats patients with chronic conditions and diseases by monitoring day-to-day health so that preventive and emergency care can be delivered as needed. With this technology matures and gains acceptance, remote medical monitoring will become the standard procedure for managing certain conditions, including heart disease and diabetes [1].

A number of different research projects are exploring medical sensor networks. Most projects are concerned with developing wearable medical sensors, while others have developed infrastructures for monitoring individual patients during daily activity, at home, or in hospitals. Paper [2] can be considered as a landmark summary of telemedicine. In this paper, the emerging wireless and networking technologies and their use to promote the ultimate goal of global health by means of deployment of a telemedicine paradigm is discussed. Authors conduct a state of-the-art study of recent wireless and medical sensor technologies in conjunction with investigation of growing pressures for a better health care service throughout the world triggered by the growing age population, social transformations. However, for as much as this, more general concept other than WSN based Health care system is discussed in this paper. According to author's point of view, sensor networks can be a very important part of the next generation of wireless computing for building a new harmonized health care infrastructure. Lin et al. used a personal digital assistant (PDA) to monitor and collect the physiological parameters which are immediately transmitted via wireless local area networks (WLAN) to be analyzed by medical personnel [4]. Rasid and Woodward used Bluetooth as wireless communication device to communicate with a remote central management unit [5].

The paper deals with the monitoring system based on ZigBee wireless sensor network technique in details. The system adopts single-chipped microprocessor ZIC2410 radio frequency chip. By the upper computer software and programs of various physiological data acquisition, the physiological data of warded people, such as the pulse, blood pressure, blood oxygen saturation and electrocardiogram, are gathered by the PC software in real time, and then the collected data are transmitted to the remote medical monitoring center through the wireless sensor network. The data information of each node, including the location and health status of the warded people, can be displayed by the monitoring center software.

\section{System Design}

This paper adopts ZigBee wireless sensor network technology to design remote health monitoring 
system. The hardware platform consists of ZigBee sensor nodes and base station equipment.

Transmitting data from sensors to medical monitoring base station equipment through wireless transmission module, and then to other networks by wireless terminals. At the same time, data are sent to the remote monitoring centre through Internet network to be statistical analyzed by professional staff. Then necessary advisory services and medical advice can be provided, so the actual realization of tele-monitoring medical is implemented.

Patient-related information (physiological data) are transferred via wireless, the patients can received immediate care and guidance from the monitoring center.

In this way, it greatly reduces or eliminates toing and froing of patients between in homes and hospitals. This system has a relatively strong usability and economy. Related settings can be implemented according to the different situations, so the system is very operational, flexible, powerful, portable and strong.

At the same time, because the system data is transmitted by wireless way, it is easier to extend and more suitable for medium and large health systems patient monitoring and medical staff and management and so on. Then it can find wide application in the future.

\section{System Structure}

From the perspective of computer information science, Remote medical system is composed of software applications and network parameter. Network environment is fundamental to assure sending the patient related information, especially for correct transmission of medical images. Remote medical network structure now generally uses the 1nternet/Intranet schema, including consulting experts, online hospital and online consultation Center, as shown in Fig. 1.

Remote medical monitoring system based on WSN is used to supplement the existing health care system, improve the ward environment, reduce the workload of medical staff, and improve the efficiency and quality of service to patients. A wireless sensing guardianship network is built using ZigBee technology constitute in the system, in which the sensor nodes collect data and images that patients needed to measure, such as: ECG, and pulse, and breathing, and temperature, the information is sent and received through ZigBee module in order to achieved data by sensor module to medical guardianship station of transmission. Then the wireless module Wi-Fi of terminal transmits data to LAN to link the system to Internet network. Later data are sent transmission to health care centers by Internet Network. After making statistics, observations and analysis of date by experts, patients can be provided with accurate medical guidance and appropriate treatment.

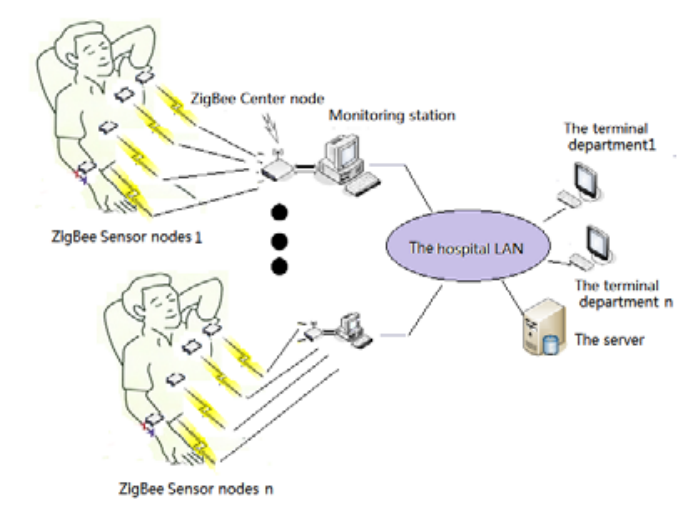

Fig.1. Remote medical monitoring system diagram

\section{System Composition}

The system consists of ECG sensor, temperature senso , breathing sensor and ZigBee modules. This paper focuses on gathering ECG signals and temperature information and assisted respiration signal for expert to analyze the patient's condition. Patient testing signal is achieved by the appropriate medical sensor. 
These sensors are pasted on the corresponding parts of the patient's body to collected body signal. Then signals are adjusted by circuit, which are amplified, processed and conversed from the analog to digital, and then they are sent to network router with ZigBee module by wireless transmission.

Signal transmission process is described as follows. Date is collected by wireless sensor module of the node. After the dates are processed through monitoring compute, the date is sent by ZigBee wireless module in electromagnetic waves of forms. In another end of the system, once wireless receiver module detect the signals, it will quickly receive date After being processed, data will be sent to PC through RS-232 serial interface or through wireless module (Wi-Fi module) will in order to achieve remote control.

ZigBee technology is used for electronic data transmission shown in Fig.2

Hardware structure and software flow chart of the system are shown in Fig.3-5

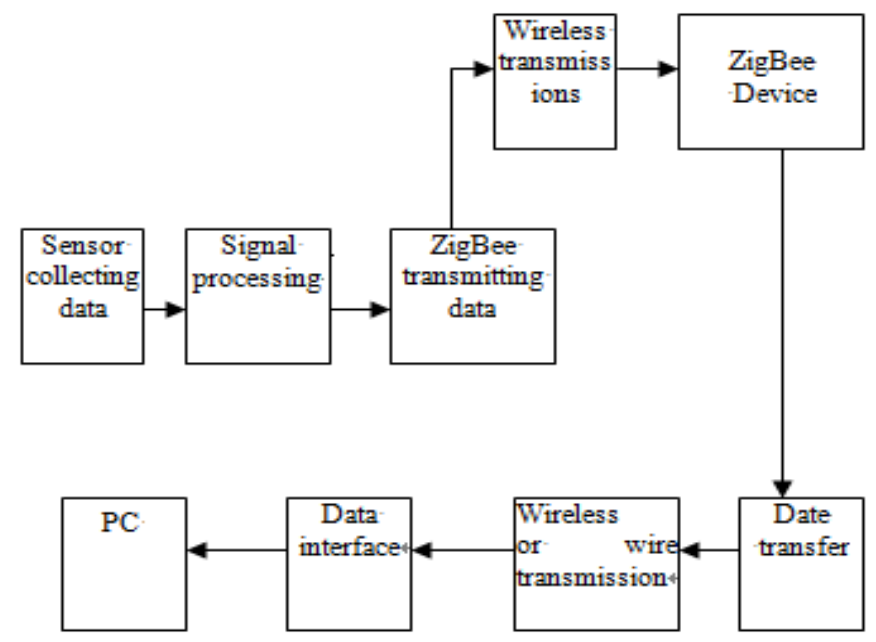

Fig.2. Electronic data transmission based on ZigBee

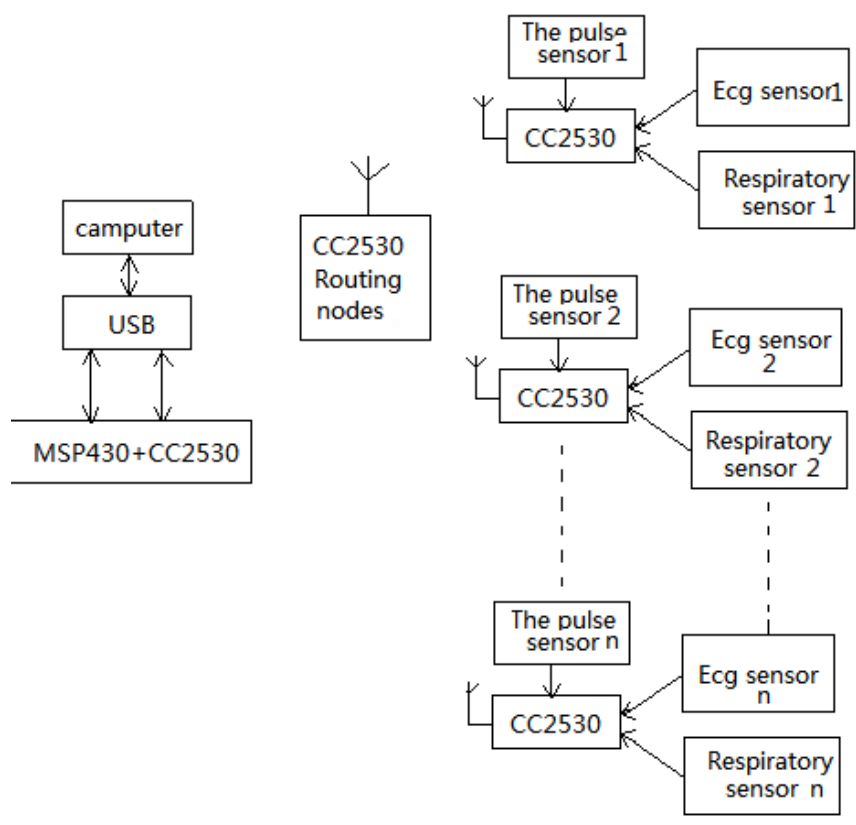

Fig.3. System hardware structure 


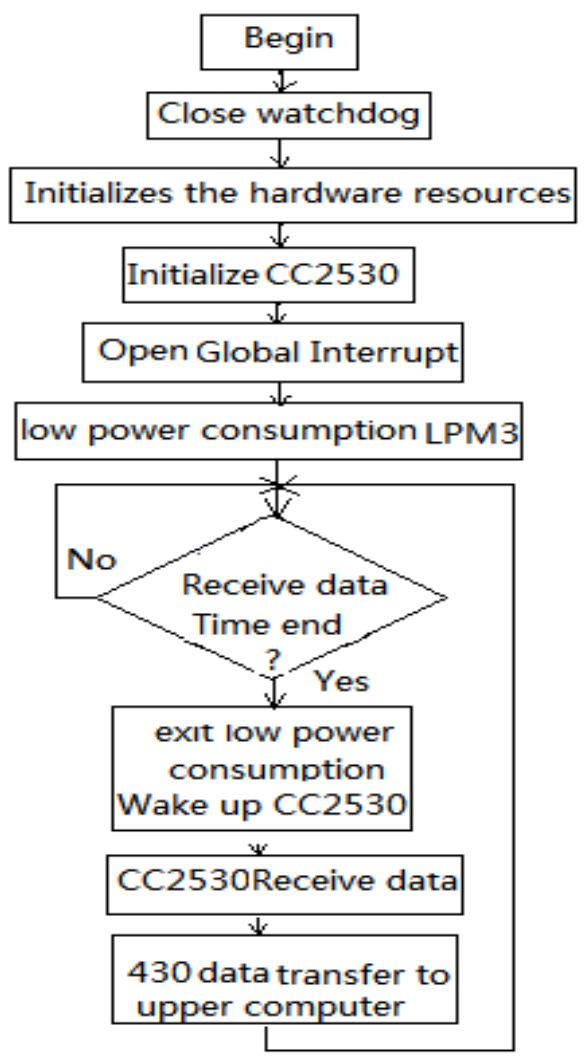

Fig.4. Software flow chart of system

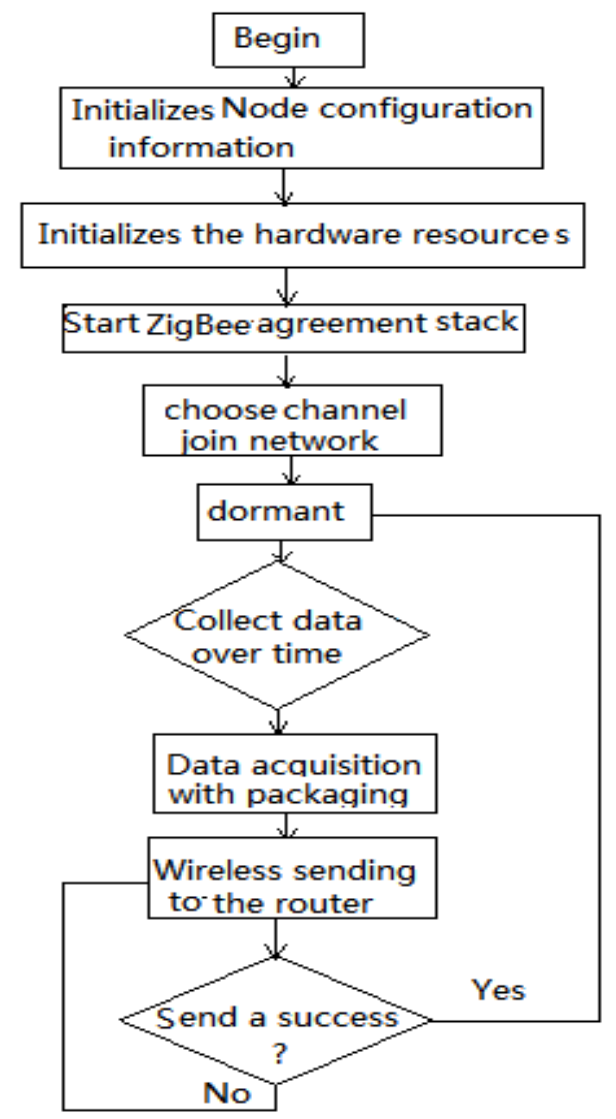

Fig.5. Software flow chart of system 


\section{Software Design of host computer}

System software design of host computer monitoring system uses LabVIEW virtual instrument software. LabVIEW is a graphical programming language used structures, also known as the "g" language in terms of data acquisition and instrument control. LabVIEW provides a number of display object or control to meet the testing requirements for measurement and control of different areas, such as knobs, tables, charts, buttons, etc, which greatly facilitates maintenance and software development work.

Monitoring curves are shown in Fig. 6-8, which are ECG, respiratory rate and pulse signal of the human body. The doctor can easily obtain real-time status of the patient through the acquisition of these signals. Selected historical data in the chart, doctor can determine the patient's changing conditions based on current data, so it is very conducive for doctor to achieve fast and efficient diagnosis of the patient.

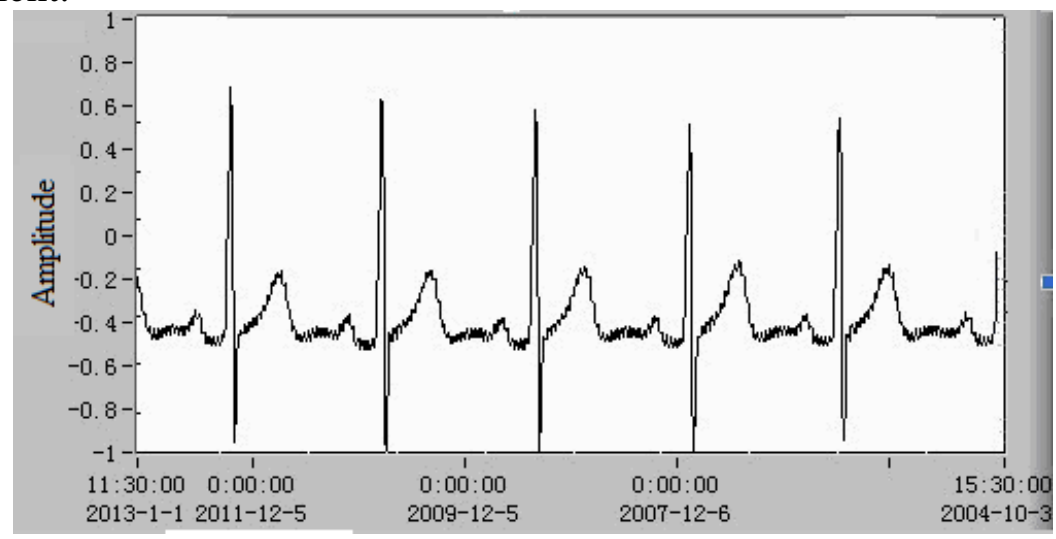

Fig.6. ECG signal

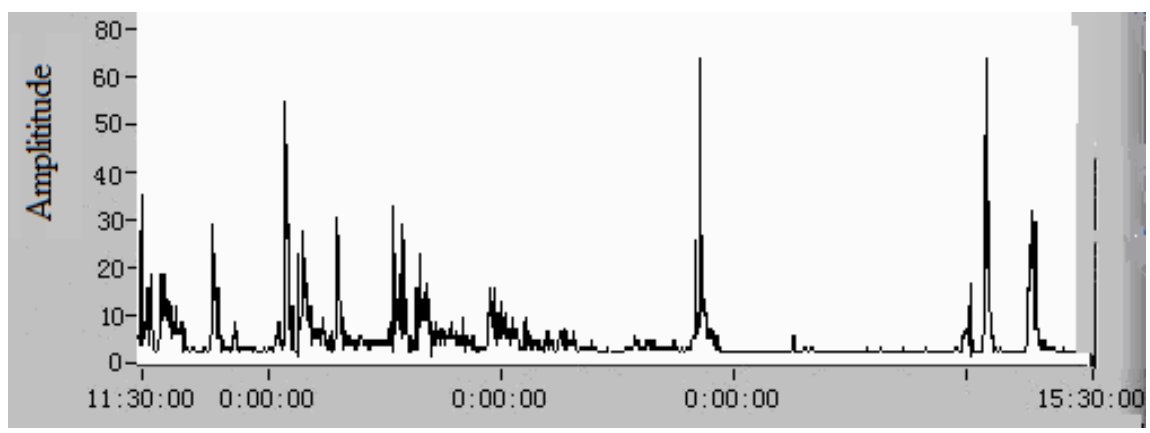

Fig.7. Respiratory rate

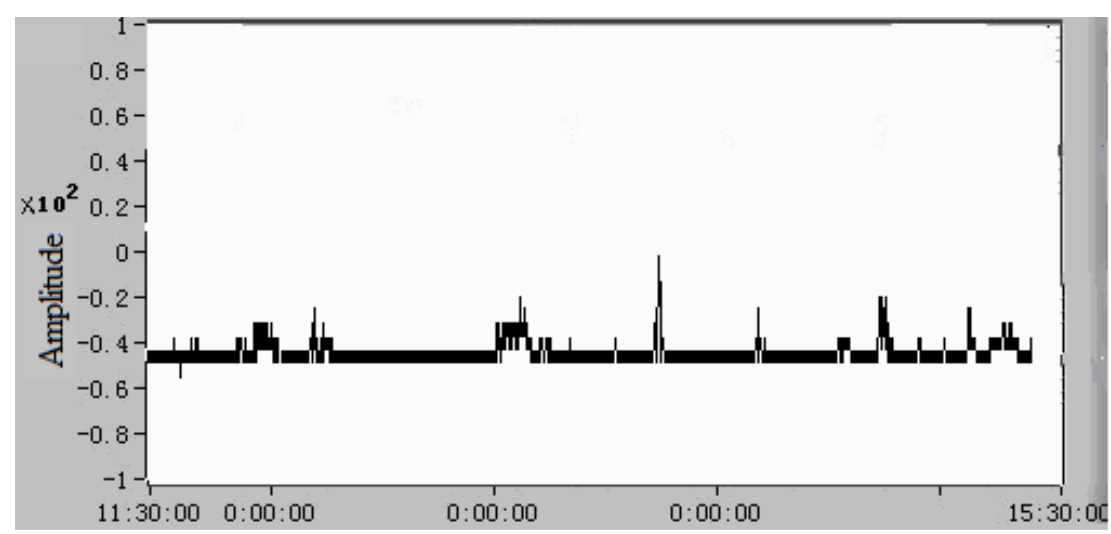

Fig.8. Pulse signal 


\section{Conclusion}

Remote medical monitoring system is designed based on the technology of wireless sensor networks and ZigBee. The system uses the ZIC2410 and LabVIEW software. The system first collects patient data and dynamically monitors patients, and then experts make analysis and diagnosis through the data coming from the network.

The whole system has low power consumption, strong portability and extensibility. This subject used advanced technology-wireless sensor network to build wireless remote health guardianship system, it will collect ECG signals, and breathing and pulse signal to achieve guardianship.

The complete of system is applied to hospital patients and non-hospital patients to achieve real-time acquisition physiological data of all patients. Data transmission uses network to get automatic records management and smart guardianship treatment. The system reduces the patients' medical program and the cumbersome process of medical workers gathering data at maximum extent. It completely changes the current hospital Manual of ECG, respiration, pulse and other data measurement method, which effectively improve the efficiency and quality of work of medical staff.

\section{References}

[1] Jurik, A.D., Weaver, A.C. Remote Medical Monitoring Compute r[J], 2008:41(4) 96 -99.

[2] H. F. Rashvand, V. T. Salcedo, E. M. Sanchez, D. Iliescu. Ubiquitous wireless telemedicine [J], IET Communications, 2008:2(2) 237-254.

[3] A. I. Heernandez, F. Mora, G. Vollegas, G. Passariello, G. Carrault, Real-time ECG transmission via Internet for nonclinical applications[J], IEEE Trans. Inf. Technol. Biomed. 2001:5(3) 253-257.

[3] Y.-H. Lin, I.-C Jan, P. C.-I. Ko, Y.-Y. Chen, J.-M. Wong, G.-J. Jan. A wireless PDA-based physiological monitoring system for patient transport [J], IEEE Trans. Inf. Technol. Biomed, 2004:8(4) 439-447.

[4] M.F.A Rasid, B. Woodward, Bluetooth telemedicine processor for multichannel biomedical monitoring via mobile cellular networks [J], IEEE Trans. Inf. Technol. Biomed., 2005:9(1) 35-43.

[5] Hairong Yan, Hongwei Huo, Youzhi Xu, Gidlund, M. Wireless sensor network based E-health system implementation and experimental results [J], IEEE Transactions on Consumer Electronics, 2010: 56 (4) 2288-2295.

[6] Song Aijuan, Study on Remote Medical System Based on Wireless Sensor Network, Dissertation in Biomedical Engineering [D], Yanshan University. 